Bull. Korean Math. Soc. 50 (2013), No. 6, pp. 1873-1886

http://dx.doi.org/10.4134/BKMS.2013.50.6.1873

\title{
INNER UNIFORM DOMAINS AND THE APOLLONIAN INNER METRIC
}

\author{
YAXiAng Li AND XiantaO WANG
}

\begin{abstract}
In this paper, we characterize inner uniform domains in $\mathbb{R}^{n}$ in terms of Apollonian inner metric and the metric $j_{D}^{\prime}$ when $D$ are Apollonian. As an application, a new characterization for $A$-uniform domains is obtained.
\end{abstract}

\section{Introduction and main results}

Throughout the paper, we assume that $D$ is a proper subdomain of the Euclidean $n$-space $\mathbb{R}^{n}, n \geq 2,[x, y]$ denotes the closed segment between $x$ and $y$, and $B^{n}(x, r)$ stands for the open ball centered at $x$ with radius $r>0$, i.e., $B^{n}(x, r)=\left\{y \in \mathbb{R}^{n}:|y-x|<r\right\}$. In particular, we use $\mathbb{B}^{n}$ to denote the unit ball $B^{n}(0,1)$. For $x, y \in D$, the Apollonian distance is defined by

$$
\alpha_{D}(x, y)=\sup _{a, b \in \partial D}\left\{\log \frac{|a-x||b-y|}{|a-y||b-x|}\right\},
$$

where $\partial D$ means the boundary of $D$. If one of $a, b$ equals to $\infty$, we understand that $\frac{|\infty-x|}{|\infty-y|}=1$. We note that this metric is invariant under Möbius transformations and equals the hyperbolic distance in balls and half spaces (cf. [2]). It is in fact a metric if and only if the complement of $D$ is not contained in a hyperplane as was noted in [2, Theorem 1.1] (see also [9]). In this paper, these domains are called to be Apollonian. This metric was introduced in [2] and considered in $[1,5,8,9,10,11,12,19,20]$.

Let $\gamma:[0,1] \rightarrow D$ be a path, i.e., a continuous function. If $d$ is a metric in $D$, then the $d$-length of $\gamma$ is defined by

$$
d(\gamma)=\sup \left\{\sum_{i=0}^{k-1} d\left(\gamma\left(t_{i}\right), \gamma\left(t_{i+1}\right)\right)\right\}
$$

Received April 6, 2012; Revised May 18, 2013.

2010 Mathematics Subject Classification. Primary 30C65, 30F45; Secondary 30C20.

Key words and phrases. inner uniform, Apollonian metric, inner metric.

The research was partly supported by NSF of China (No. 11071063) and Hunan Provincial Innovation Foundation For Postgraduate. 
where the supremum is taken over all $k<\infty$ and all sequences $\left\{t_{i}\right\}$ satisfying $0=t_{0}<t_{1}<\cdots<t_{k}=1$. All the paths in this paper are assumed to be rectifiable, that is, they have the finite Euclidean arc length. The inner metric of $d$ is defined by the formula

$$
\widetilde{d}(x, y)=\inf _{\gamma}\{d(\gamma)\}
$$

where the infimum is taken over all paths connecting $x$ and $y$ in $D$. Particularly, we use $\widetilde{\alpha}_{D}$ to denote the inner metric of the Apollonian metric $\alpha_{D}$ and call it the Apollonian inner metric. Also we use $\lambda_{D}(x, y)$ to denote $\widetilde{d}(x, y)$ when $d(\gamma)$ is the Euclidean arc length.

In [8, Theorem 1.2], Hästö proved that $\widetilde{\alpha}_{D}$ is a metric if and only if the complement of $D$ is not contained in an $(n-2)$-dimensional hyperplane in $\mathbb{R}^{n}$. Further, in [8], Hästö showed:

Theorem A ([8, Theorem 1.5]). Let $D$ be Apollonian. Then for $x, y \in D$, there exists a path $\gamma$ in $D$ connecting $x$ and $y$ such that

$$
\alpha_{D}(\gamma)=\widetilde{\alpha}_{D}(x, y) \text {. }
$$

And further, in [13], the authors got the following.

Theorem B ([13, Lemma 2.4]). Let $x, y \in D$ and let $\gamma \subset D$ be a path such that $\widetilde{\alpha}_{D}(x, y)=\alpha_{D}(\gamma)$. Then for each $z, w \in \gamma$, we have

$$
\widetilde{\alpha}_{D}(z, w)=\alpha_{D}(\gamma[z, w])
$$

where $\gamma[z, w]$ denotes the part of $\gamma$ between $z$ and $w$.

Definition 1. A domain $D$ is called inner c-uniform provided there exists a positive constant $c$ such that each pair of points $z_{1}, z_{2}$ in $D$ can be joined by a rectifiable arc $\gamma$ in $D$ satisfying (cf. [24])

(1) $\min \left\{\ell\left(\gamma\left[z_{1}, z\right]\right) \ell\left(\gamma\left[z_{2}, z\right]\right)\right\} \leq c d_{D}(z)$ for all $z \in \gamma$; and

(2) $\ell(\gamma) \leq c \lambda_{D}\left(z_{1}, z_{2}\right)$

where $d_{D}(z)$ denotes the distance from $z$ to the boundary $\partial D$ of $D$.

If $\lambda_{D}\left(z_{1}, z_{2}\right)$ is replaced by $\left|z_{1}-z_{2}\right|$ in Definition 1 , then $D$ is said to be c-uniform.

Obviously, uniformity implies inner uniformity.

Definition 2. A domain $D$ is called to be a $c$-John domain provided there exists a positive constant $c$ such that each pair of points $z_{1}, z_{2}$ in $D$ can be joined by a rectifiable arc $\gamma$ in $D$ satisfying (cf. [18])

$$
\min \left\{\operatorname{diam}\left(\gamma\left[z_{1}, w\right]\right), \operatorname{diam}\left(\gamma\left[w, z_{2}\right]\right)\right\} \leq c d_{D}(w) .
$$

In [24], Väisälä showed the following two theorems.

Theorem C ([24, Theorem 3.3 and Theorem 3.4]). Suppose that $D \subset \mathbb{R}^{n}$ is an inner c-uniform domain. Then for $x, y \in D$, we have

$$
\lambda_{D}(x, y) \leq \nu_{1} \varrho_{D}(x, y),
$$


where $\nu_{1} \geq 6 c$ is a constant depending on $c$ and $n$, and $\varrho_{D}(x, y)$ denotes the inner diameter metric, defined by

$$
\varrho_{D}(x, y)=\inf _{\gamma}\{\operatorname{diam}(\gamma)\}
$$

over all arcs $\gamma$ joining $x$ and $y$ in $D$.

Theorem D ([24, Theorem 3.11]). For a domain $D \subset \mathbb{R}^{n}$, the following conditions are quantitatively equivalent:

(1) $D$ is inner c-uniform.

(2) Each pair of points $z_{1}, z_{2} \in D$ can be joined by an arc $\gamma$ such that for $w \in \gamma$,

$\min \left\{\operatorname{diam}\left(\gamma\left[z_{1}, w\right]\right), \operatorname{diam}\left(\gamma\left[z_{2}, w\right]\right)\right\} \leq \nu_{2} d_{D}(w)$ and $\operatorname{diam}(\gamma) \leq \nu_{2} \varrho_{D}\left(z_{1}, z_{2}\right)$, where the constants $c$ and $\nu_{2}$ depend on each other and $n$.

Let $D$ be a domain and $x, y \in D$. We write

$$
j_{D}(x, y)=\log \left(1+\frac{|x-y|}{\min \left\{d_{D}(x), d_{D}(y)\right\}}\right) .
$$

Kim [14] (see also [24]) introduced the following version of the $j$-metric:

$$
j_{D}^{\prime}(x, y)=\log \left(1+\frac{\varrho_{D}(x, y)}{\min \left\{d_{D}(x), d_{D}(y)\right\}}\right),
$$

and the quasihyperbolic metric [7] is defined by

$$
k_{D}(x, y)=\inf _{\gamma} \int_{\gamma} \frac{|d z|}{d_{D}(z)}
$$

where the infimum is taken over all paths $\gamma$ joining $x$ and $y$ in $D$.

We easily know from the proof of [22, Lemma 2.2] that for $x, y \in D$,

$$
j_{D}(x, y) \leq j_{D}^{\prime}(x, y) \leq k_{D}(x, y) \text {. }
$$

Further, we have:

Theorem E. For $x, y \in D$, the following hold true.

(1) $\left(\left[2\right.\right.$, Corollary 3.2]) $\quad\left|\log \frac{d_{D}(x)}{d_{D}(y)}\right| \leq \alpha_{D}(x, y) \leq 2 j_{D}(x, y)$;

(2) $\left(\left[9\right.\right.$, Lemma 5.3]) $\widetilde{j}_{D}(x, y)=k_{D}(x, y)$;

(3) $\left(\left[9\right.\right.$, Corollary 5.4]) $\quad \widetilde{\alpha}_{D}(x, y) \leq 2 k_{D}(x, y)$.

In [6], Gehring and Osgood got a characterization of uniform domains in terms of $k_{D}$ and $j_{D}$.

Theorem F ([6, Corollary 1]). A domain $D$ is $\mu$-uniform if and only if there exists a constant $\mu_{1}$ such that

$$
k_{D}\left(z_{1}, z_{2}\right) \leq \mu_{1} j_{D}\left(z_{1}, z_{2}\right)
$$

for all $z_{1}, z_{2} \in D$, where the constants $\mu$ and $\mu_{1}$ depend only on each other. 
As a matter of fact, the above inequality appearing in [6] in a form with an additive constant on the right hand side: it was shown by Vuorinen [25, 2.50] that the additive constant can be chosen to be 0 . Moreover, in [13], the authors proved the following.

Theorem G ([13, Theorem 1.2]). A domain $D \subset \mathbb{R}^{n}$ is $\mu$-uniform if and only if there exists a constant $\mu_{2}$ such that $\widetilde{\alpha}_{D}(x, y) \leq \mu_{2} j_{D}(x, y)$ for any $x, y \in D$, where the constants $\mu$ and $\mu_{2}$ depend only on each other.

See $[3,4,6,13,15,16,17,24,21,23]$ for more details on uniform domains and inner uniform domains.

By Theorem G, one may ask that if we can characterize inner uniform domains in terms of $\widetilde{\alpha}_{D}$ and $j_{D}^{\prime}$. The main aim of this paper is to consider this problem. Our result shows that the answer to this problem is affirmative. Combining with [15, Theorem 2.1] and Theorem D, we state our result in the following form.

Theorem 1. Let $D$ be a proper subdomain of $\mathbb{R}^{n}$. If $D$ is Apollonian, then the followings are quantitatively equivalent.

(1) $D$ is an inner c-uniform domain;

(2) There exists a constant $c_{1}$ such that

$$
k_{D}(x, y) \leq c_{1} j_{D}^{\prime}(x, y) \quad \forall x, y \in D ;
$$

(3) There exists a constant $c_{2}$ such that

$$
\widetilde{\alpha}_{D}(x, y) \leq c_{2} j_{D}^{\prime}(x, y) \quad \forall x, y \in D ;
$$

(4) Each pair of points $x, y \in D$ can be joined by an arc $\gamma$ such that for $w \in \gamma, \min \{\operatorname{diam}(\gamma[x, w]), \operatorname{diam}(\gamma[y, w])\} \leq c_{3} d_{D}(w)$ and $\operatorname{diam}(\gamma) \leq$ $c_{3} \varrho_{D}(x, y)$,

where $c, c_{1}, c_{2}$ and $c_{3}$ are constants greater than 1 , and depend on each other and $n$.

In [9], Hästö proved the following result.

Theorem H ([9, Proposition 6.6]). Let $D \subset \mathbb{R}^{n}$ be a domain. The following conditions are quantitatively equivalent:

(1) $D$ is A-uniform with coefficient $K$, that is, there exist some constant $K$ such that for $x, y \in D, k_{D}(x, y) \leq K \alpha_{D}(x, y)$;

(2) $D$ is $\mu$-uniform and has the comparison property with some constant $L$;

(3) $D$ is $\mu_{3}$-quasi-isotropic and $\widetilde{\alpha}_{D} \leq \mu_{4} \alpha_{D}$,

where the constants $K, L, \mu, \mu_{3}$ and $\mu_{4}$ depend only on each other.

Here we say that a domain $D \subset \mathbb{R}^{n}$ has the comparison property if there exists a constant $L$ such that

$$
j_{D} / L \leq \alpha_{D} \leq 2 j_{D}
$$


and $D$ is $\mu_{3}$-quasi-isotropic if

$$
\limsup _{r \rightarrow 0} \frac{\sup \left\{\alpha_{D}(x, z):|x-z|=r\right\}}{\inf \left\{\alpha_{D}(x, y):|x-y|=r\right\}} \leq \mu_{3}
$$

for every $x \in D$ (see [9]).

As an application of Theorem 1, we get a new characterization for $A$-uniform domains.

Corollary 1. Let $D \subset \mathbb{R}^{n}$ be an Apollonian domain. The following conditions are quantitatively equivalent:

(1) $D$ is A-uniform with coefficient $K$;

(2) $D$ is c-inner uniform and $j_{D}^{\prime}(x, y) \leq \mu_{5} \alpha_{D}(x, y)$ for all $x, y \in D$, where the constants $c, K$ and $\mu_{5}$ depend on each other and $n$.

In the next section, we will prove Theorem 1 and Corollary 1.

\section{Proofs of Theorem 1 and Corollary 1}

\subsection{Proof of Theorem 1}

The implication $(1) \Rightarrow(2)$ follows from [15, Theorem 2.1] and Theorem C, and Theorem E shows that $(2) \Rightarrow(3)$ is true. The implication $(4) \Rightarrow(1)$ follows from Theorem D. Hence to finish the proof of Theorem 1, it remains only one implication $(3) \Rightarrow(4)$ to be checked.

Suppose that the assertion (3) in the theorem holds. To prove the truth of the assertion of (4) in the theorem, we let $x, y \in D$. Without loss of generality, we assume that $d_{D}(x) \leq d_{D}(y)$. We consider the case where $|x-y|<d_{D}(x)$ and the case where $|x-y| \geq d_{D}(x)$, separately.

Case 1. $|x-y|<d_{D}(x)$.

Let $\gamma=[x, y]$ be the Euclidean line segment joining $x$ and $y$. Clearly, $\gamma \subset D$,

$$
\operatorname{diam}(\gamma)=|x-y|=\varrho_{D}(x, y)
$$

and

$$
\min \{\operatorname{diam}(\gamma[x, w]), \operatorname{diam}(\gamma[y, w])\} \leq d_{D}(w)
$$

for $w \in \gamma$. Thus the assertion (4) in the theorem is true in this case.

Case 2. $|x-y| \geq d_{D}(x)$.

By Theorem A there exists a path $\gamma \subset D$ connecting $x$ and $y$ such that

$$
\widetilde{\alpha}_{D}(x, y)=\alpha_{D}(\gamma)
$$

By compactness we see that there is a point $z_{0}$ in $\gamma$ which is the first point along the direction from $x$ to $y$ satisfying

$$
d_{D}\left(z_{0}\right)=\sup _{w \in \gamma}\left\{d_{D}(w)\right\}
$$


Let $m \geq 0$ be the integer such that

$$
2^{m} d_{D}(x) \leq d_{D}\left(z_{0}\right)<2^{m+1} d_{D}(x),
$$

and let $x_{0}$ be the first point of $\gamma\left[x, z_{0}\right]$ from $x$ to $z_{0}$ with

$$
d_{D}\left(x_{0}\right)=2^{m} d_{D}(x) .
$$

Then we have

$$
d_{D}\left(x_{0}\right) \leq d_{D}\left(z_{0}\right)<2 d_{D}\left(x_{0}\right) .
$$

Let $x_{1}=x$, and let $x_{2}, \ldots, x_{m+1}$ be the points such that for each $i \in\{2, \ldots, m+$ $1\}, x_{i}$ denotes the first point in $\gamma\left[x, z_{0}\right]$ along the direction from $x$ to $z_{0}$ satisfying

$$
d_{D}\left(x_{i}\right)=2^{i-1} d_{D}\left(x_{1}\right) .
$$

Apparently, $x_{m+1}=x_{0}$. If $x_{0} \neq z_{0}$, we denote $z_{0}$ by $x_{m+2}$. By the choice of $x_{i}$, we know that for each $i \in\{1,2, \ldots, m\}$,

$$
d_{D}\left(x_{i+1}\right)=2 d_{D}\left(x_{i}\right),
$$

and so

$$
\varrho_{D}\left(x_{i}, x_{i+1}\right) \geq d_{D}\left(x_{i+1}\right)-d_{D}\left(x_{i}\right)=d_{D}\left(x_{i}\right) .
$$

For each $i \in\{1,2, \ldots, m\}$ and $w \in \gamma\left[x_{i}, x_{i+1}\right]$, it easily follows that

$$
d_{D}(w) \leq d_{D}\left(x_{i+1}\right)=2 d_{D}\left(x_{i}\right) .
$$

Let $w_{0}$ be the first point of $\gamma$ along the direction from $y$ to $x$ satisfying

$$
d_{D}\left(w_{0}\right)=\sup _{w \in \gamma}\left\{d_{D}(w)\right\}
$$

Obviously, $d_{D}\left(w_{0}\right)=d_{D}\left(z_{0}\right)$. It is possible that $w_{0}=z_{0}$. A similar argument as above shows that there are points $\left\{y_{j}\right\}_{j=1}^{s+1}$ in $\gamma\left[y, w_{0}\right]$ such that for each $j \in\{1, \ldots, s+1\}, y_{j}$ denotes the first point in $\gamma\left[y, w_{0}\right]$ along the direction from $y$ to $w_{0}$ satisfying

$$
d_{D}\left(y_{j}\right)=2^{j-1} d_{D}\left(y_{1}\right),
$$

where $y_{1}=y$ and $d_{D}\left(y_{s+1}\right)=2^{s} d_{D}\left(y_{1}\right)$. We also use $y_{0}$ to denote $y_{s+1}$. If $y_{0} \neq w_{0}$, we use $y_{s+2}$ to denote $w_{0}$.

Lemma 1. For each $i \in\{1,2, \ldots, m\}$, we have

(1) $\operatorname{diam}\left(\gamma\left[x_{i}, x_{i+1}\right]\right) \leq b_{1} \varrho_{D}\left(x_{i}, x_{i+1}\right)$ with $b_{1}=24 c_{2}^{\prime}$ and $c_{2}^{\prime}=\left[c_{2}\right]+1$. Here and in the following, [.] always denotes the greatest integer part;

(2) $\varrho_{D}\left(x_{i}, x_{i+1}\right) \leq b_{2} d_{D}\left(x_{i}\right)$ with $b_{2}=\left(1+b_{1}\right)^{2}$;

(3) $d_{D}\left(x_{i}\right) \leq b_{3} d_{D}(w)$ for all $w \in \gamma$ with $b_{3}=\left(1+b_{2}\right)^{\frac{c_{2}}{2}}$, where $c_{2}$ is the same constant as in the inequality (1.2). 
Proof. We now prove the first assertion in the lemma. Suppose on the contrary that there is some $i \in\{1, \ldots, m\}$ satisfying

$$
\operatorname{diam}\left(\gamma\left[x_{i}, x_{i+1}\right]\right)>b_{1} \varrho_{D}\left(x_{i}, x_{i+1}\right) .
$$

Let $u_{i, 1}=x_{i}$, and take the points $u_{i, 2}, u_{i, 3}, \ldots, u_{i, c_{2}^{\prime}+1}$ in $\gamma$ such that for each $t \in\left\{2, \ldots, c_{2}^{\prime}+1\right\}, u_{i, t}$ is the first point of $\gamma$ from $x_{i}$ to $x_{i+1}$ satisfying

$$
\left|x_{i}-u_{i, t}\right|=6(t-1) \varrho_{D}\left(x_{i}, x_{i+1}\right) .
$$

Then for each $t \in\left\{1, \ldots, c_{2}^{\prime}\right\}$, we have

$$
\left|u_{i, t}-u_{i, t+1}\right| \geq\left|x_{i}-u_{i, t+1}\right|-\left|u_{i, t}-x_{i}\right| \geq 6 \varrho_{D}\left(x_{i}, x_{i+1}\right) .
$$

Let $p \in \partial D$ be such that $d_{D}\left(u_{i, t+1}\right)=\left|u_{i, t+1}-p\right|$. Then (2.4), (2.5) and (2.6) yield

$$
\begin{aligned}
\left|u_{i, t}-p\right| & \geq\left|u_{i, t}-u_{i, t+1}\right|-d_{D}\left(u_{i, t+1}\right) \\
& \geq 6 \varrho_{D}\left(x_{i}, x_{i+1}\right)-2 d_{D}\left(x_{i}\right) \\
& \geq 2 \varrho_{D}\left(x_{i}, x_{i+1}\right)+2 d_{D}\left(x_{i}\right) .
\end{aligned}
$$

Similarly, for $q \in \partial D$ with $d_{D}\left(u_{i, t}\right)=\left|u_{i, t}-q\right|$, we get

$$
\left|u_{i, t+1}-q\right| \geq 2 \varrho_{D}\left(x_{i}, x_{i+1}\right)+2 d_{D}\left(x_{i}\right) .
$$

Thus we infer from $(2.5),(2.7)$ and (2.8) that

$$
\alpha_{D}\left(u_{i, t}, u_{i, t+1}\right) \geq \log \left(\frac{\left|u_{i, t}-p\right|}{d_{D}\left(u_{i, t+1}\right)} \frac{\left|u_{i, t+1}-q\right|}{d_{D}\left(u_{i, t}\right)}\right) \geq 2 \log \left(1+\frac{\varrho_{D}\left(x_{i}, x_{i+1}\right)}{d_{D}\left(x_{i}\right)}\right),
$$

which together with Theorem B show that

$$
\begin{aligned}
\widetilde{\alpha}_{D}\left(x_{i}, x_{i+1}\right) & =\alpha_{D}\left(\gamma\left[x_{i}, x_{i+1}\right]\right) \\
& \geq \sum_{t=1}^{c_{2}^{\prime}} \alpha_{D}\left(u_{i, t}, u_{i, t+1}\right) \\
& \geq 2 c_{2}^{\prime} \log \left(1+\frac{\varrho_{D}\left(x_{i}, x_{i+1}\right)}{d_{D}\left(x_{i}\right)}\right) \\
& \geq 2 c_{2} \log \left(1+\frac{\varrho_{D}\left(x_{i}, x_{i+1}\right)}{d_{D}\left(x_{i}\right)}\right) \\
& =2 c_{2} j_{D}^{\prime}\left(x_{i}, x_{i+1}\right),
\end{aligned}
$$

which contradicts with (1.2). Hence (1) is true.

Then we come to prove the second assertion. Suppose on the contrary that there is some $i \in\{1,2, \ldots, m\}$ satisfying

$$
\varrho_{D}\left(x_{i}, x_{i+1}\right)>b_{2} d_{D}\left(x_{i}\right) .
$$

Obviously, there exists some point $v \in \gamma\left[x_{i}, x_{i+1}\right]$ such that $\left|x_{i}-v\right| \geq$ $\frac{1}{2} \varrho_{D}\left(x_{i}, x_{i+1}\right)$. We let $v_{i, 1}=x_{i}$, and let $v_{i, 2}, \ldots, v_{i, \frac{b_{1}}{12}+1}$ be the points in $\gamma$ 
such that for each $h \in\left\{2, \ldots, \frac{b_{1}}{12}+1\right\}, v_{i, h}$ is the first point of $\gamma$ from $x_{i}$ to $x_{i+1}$ satisfying

$$
\left|x_{i}-v_{i, h}\right|=\frac{6(h-1)}{b_{1}} \varrho_{D}\left(x_{i}, x_{i+1}\right) .
$$

Then

$$
\left|v_{i, h}-v_{i, h+1}\right| \geq\left|v_{i, h+1}-x_{i}\right|-\left|v_{i, h}-x_{i}\right| \geq \frac{6}{b_{1}} \varrho_{D}\left(x_{i}, x_{i+1}\right) .
$$

Let $p \in \partial D$ satisfy $d_{D}\left(v_{i, h+1}\right)=\left|v_{i, h+1}-p\right|$. Then it follows from (2.4), (2.5), (2.9) and (2.10) that

$$
\begin{aligned}
\left|v_{i, h}-p\right| & \geq\left|v_{i, h}-v_{i, h+1}\right|-d_{D}\left(v_{i, h+1}\right) \\
& \geq \frac{6}{b_{1}} \varrho_{D}\left(x_{i}, x_{i+1}\right)-2 d_{D}\left(x_{i}\right) \\
& >\frac{2}{b_{1}} \varrho_{D}\left(x_{i}, x_{i+1}\right)+2 d_{D}\left(x_{i}\right) .
\end{aligned}
$$

Similarly, for $q \in \partial D$ with $d_{D}\left(v_{i, h}\right)=\left|v_{i, h}-q\right|$, we know

$$
\left|v_{i, h+1}-q\right| \geq \frac{2}{b_{1}} \varrho_{D}\left(x_{i}, x_{i+1}\right)+2 d_{D}\left(x_{i}\right) .
$$

Thus we infer from $(2.5),(2.9),(2.11)$ and (2.12) that

$$
\begin{aligned}
\alpha_{D}\left(v_{i, h}, v_{i, h+1}\right) & \geq \log \left(\frac{\left|v_{i, h}-p\right|}{d_{D}\left(v_{i, h+1}\right)} \frac{\left|v_{i, h+1}-q\right|}{d_{D}\left(v_{i, h}\right)}\right) \\
& \geq 2 \log \left(1+\frac{\varrho_{D}\left(x_{i}, x_{i+1}\right)}{b_{1} d_{D}\left(x_{i}\right)}\right) \\
& >\frac{12 c_{2}}{b_{1}} \log \left(1+\frac{\varrho_{D}\left(x_{i}, x_{i+1}\right)}{d_{D}\left(x_{i}\right)}\right) .
\end{aligned}
$$

Whence Theorem B yields

$$
\begin{aligned}
\widetilde{\alpha}_{D}\left(x_{i}, x_{i+1}\right) & =\alpha_{D}\left(\gamma\left[x_{i}, x_{i+1}\right]\right) \\
& \geq \sum_{h=1}^{\frac{b_{1}}{12}} \alpha_{D}\left(v_{i, h}, v_{i, h+1}\right) \\
& >c_{2} \log \left(1+\frac{\varrho_{D}\left(x_{i}, x_{i+1}\right)}{d_{D}\left(x_{i}\right)}\right) \\
& =c_{2} j_{D}^{\prime}\left(x_{i}, x_{i+1}\right),
\end{aligned}
$$

which is the desired contradiction.

To finish the proof of Lemma 1 , it remains to check (3). Let $w \in \gamma$. Then (2) in the lemma, (1.2), Theorems B and E lead to

$$
\begin{aligned}
2 \log \frac{d_{D}\left(x_{i}\right)}{d_{D}(w)} & <\alpha_{D}\left(x_{i}, w\right)+\alpha_{D}\left(w, x_{i+1}\right) \\
& \leq \alpha_{D}\left(\gamma\left[x_{i}, x_{i+1}\right]\right)
\end{aligned}
$$




$$
\begin{aligned}
& =\widetilde{\alpha}_{D}\left(x_{i}, x_{i+1}\right) \\
& \leq c_{2} \log \left(1+\frac{\varrho_{D}\left(x_{i}, x_{i+1}\right)}{d_{D}\left(x_{i}\right)}\right) \\
& \leq c_{2} \log \left(1+b_{2}\right)
\end{aligned}
$$

which shows that

$$
d_{D}\left(x_{i}\right) \leq\left(1+b_{2}\right)^{\frac{c_{2}}{2}} d_{D}(w),
$$

which shows that $(3)$ is true by taking $b_{3}=\left(1+b_{2}\right)^{\frac{c_{2}}{2}}$. Hence the proof of Lemma 1 is complete.

Similarly, we know that:

Lemma 2. For each $j \in\{1, \ldots, s\}$, we have

(1) $\operatorname{diam}\left(\gamma\left[y_{j}, y_{j+1}\right]\right) \leq b_{1} \varrho_{D}\left(y_{j}, y_{j+1}\right)$;

(2) $\varrho_{D}\left(y_{j}, y_{j+1}\right) \leq b_{2} d_{D}\left(y_{j}\right)$

(3) $d_{D}\left(y_{j}\right) \leq b_{3} d_{D}(w)$ for all $w \in \gamma$.

Suppose $x_{0} \neq y_{0}$. Then:

Lemma 3. For $w \in \gamma\left[x_{0}, y_{0}\right]$, we have

$$
d_{D}\left(x_{0}\right) \leq b_{3}^{2} d_{D}(w) \text { and } \operatorname{diam}\left(\gamma\left[x_{0}, y_{0}\right]\right) \leq b_{4} d_{D}(w) \text {, }
$$

where $b_{4}=b_{1} b_{2} b_{3}^{2}$.

Proof. We note by (2.2) that

$$
\frac{1}{2} d_{D}\left(y_{0}\right)<d_{D}\left(x_{0}\right)<2 d_{D}\left(y_{0}\right),
$$

and for $w \in \gamma\left[x_{0}, y_{0}\right]$, we have

$$
d_{D}(w)<d_{D}\left(z_{0}\right)<2 d_{D}\left(x_{0}\right) .
$$

We prove this lemma by considering the case where $\varrho_{D}\left(x_{0}, y_{0}\right) \geq d_{D}\left(x_{0}\right)$ and the case where $\varrho_{D}\left(x_{0}, y_{0}\right)<d_{D}\left(x_{0}\right)$, separately.

Suppose first that $\varrho_{D}\left(x_{0}, y_{0}\right) \geq d_{D}\left(x_{0}\right)$. Then by (2.13), (2.14) and a similar argument as in the proof of Lemma 1 , we get for each $w \in \gamma\left[x_{0}, y_{0}\right]$,

$$
d_{D}\left(x_{0}\right) \leq b_{3}^{2} d_{D}(w)
$$

and

$$
\operatorname{diam}\left(\gamma\left[x_{0}, y_{0}\right]\right) \leq b_{4} d_{D}(w)
$$

where $b_{4}=b_{1} b_{2} b_{3}^{2}$.

Suppose next that $\varrho_{D}\left(x_{0}, y_{0}\right)<d_{D}\left(x_{0}\right)$. In this case, we need the following claim.

Claim 1. For $w \in \gamma\left[x_{0}, y_{0}\right]$, we have $\left|w-x_{0}\right| \leq\left(3^{c_{2}}+1\right) d_{D}\left(x_{0}\right)$. 
Obviously, to prove this claim, it suffices to consider the case $\left|x_{0}-w\right| \geq$ $2 d_{D}\left(x_{0}\right)$. Let $z \in \partial D$ satisfy $\left|x_{0}-z\right|=d_{D}\left(x_{0}\right)$. Then it follows from (1.2), (2.13), Theorems B and E that

$$
\begin{aligned}
\log \left(\frac{\left|w-x_{0}\right|}{d_{D}\left(x_{0}\right)}-1\right) & \leq \log \frac{|z-w|}{d_{D}\left(x_{0}\right)} \\
& \leq \alpha_{D}\left(x_{0}, w\right) \\
& \leq \alpha_{D}\left(\gamma\left[x_{0}, y_{0}\right]\right) \\
& =\widetilde{\alpha}_{D}\left(x_{0}, y_{0}\right) \\
& \leq c_{2} \log \left(1+\frac{\varrho_{D}\left(x_{0}, y_{0}\right)}{\min \left\{d_{D}\left(x_{0}\right), d_{D}\left(y_{0}\right)\right\}}\right) \\
& \leq c_{2} \log 3,
\end{aligned}
$$

from which the claim easily follows.

By Claim 1, we get

$$
\operatorname{diam}\left(\gamma\left[x_{0}, y_{0}\right]\right) \leq 2\left(3^{c_{2}}+1\right) d_{D}\left(x_{0}\right) .
$$

Moreover, by Theorem E and a similar argument as in (2.17), we also have

$$
\log \frac{d_{D}\left(x_{0}\right)}{d_{D}(w)} \leq \alpha_{D}\left(x_{0}, w\right) \leq c_{2} \log 3,
$$

and so

$$
d_{D}\left(x_{0}\right) \leq 3^{c_{2}} d_{D}(w)
$$

which together with (2.18) show that

$$
\operatorname{diam}\left(\gamma\left[x_{0}, y_{0}\right]\right) \leq 2\left(3^{c_{2}}+1\right)^{2} d_{D}(w) \leq b_{4} d_{D}(w) .
$$

The inequalities (2.15), (2.16), (2.19) and (2.20) imply that the lemma is true.

Now we come to prove that the first part of (4) in Theorem 1 holds with constant $2 b_{4}$, i.e., for $w \in \gamma$,

$$
\min \{\operatorname{diam}(\gamma[x, w]), \operatorname{diam}(\gamma[y, w])\} \leq 2 b_{4} d_{D}(w) .
$$

Let $w \in \gamma$. We divide the discussions into three cases.

Case 3. $w \in \gamma\left[x, x_{0}\right]$.

Clearly, there exists an integer $k \in\{1,2, \ldots, m\}$ such that $w \in \gamma\left[x_{k}, x_{k+1}\right]$. By Lemma 1 and (2.3) we have

$$
\begin{aligned}
\operatorname{diam}(\gamma[x, w]) & \leq \sum_{i=1}^{k} \operatorname{diam}\left(\gamma\left[x_{i}, x_{i+1}\right]\right) \leq b_{1} b_{2} \sum_{i=1}^{k} d_{D}\left(x_{i}\right) \\
& \leq 2 b_{1} b_{2} d_{D}\left(x_{k}\right) \leq 2 b_{1} b_{2} b_{3} d_{D}(w)<b_{4} d_{D}(w) .
\end{aligned}
$$

Case 4. $w \in \gamma\left[y, y_{0}\right]$. 
By Lemma 2, we see from a similar argument as in the proof of Case 3 that

$$
\operatorname{diam}(\gamma[y, w])<b_{4} d_{D}(w) .
$$

Case 5. If $w \in \gamma\left[x_{0}, y_{0}\right]$.

It follows from Lemmas 1 and 3 that

$$
\begin{aligned}
\operatorname{diam}(\gamma[x, w]) & \leq \sum_{i=1}^{m} \operatorname{diam}\left(\gamma\left[x_{i}, x_{i+1}\right]\right)+\operatorname{diam}\left(\gamma\left[x_{0}, y_{0}\right]\right) \\
& \leq b_{1} b_{2} \sum_{i=1}^{m} d_{D}\left(x_{i}\right)+b_{4} d_{D}(w) \\
& \leq b_{1} b_{2} d_{D}\left(x_{m+1}\right)+b_{4} d_{D}(w) \\
& \leq 2 b_{4} d_{D}(w) .
\end{aligned}
$$

The proof for (2.21) easily follows from the combination of $(2.22),(2.23)$ and (2.24).

Next we prove the second part of (4) in Theorem 1 with constant $b_{6}=$ $2^{b_{5}+2} b_{4}$, where $b_{5}=c_{2} \log _{2}\left(8 b_{4}+1\right)+1$, i.e.,

$$
\operatorname{diam}(\gamma[x, y]) \leq b_{6} \varrho_{D}(x, y) .
$$

We first prove a lemma.

Lemma 4. $\varrho_{D}(x, y) \geq 2^{m-b_{5}} d_{D}(x)$.

Proof. If $m \leq b_{5}$, then it is obvious from the assumption " $|x-y| \geq d_{D}(x)$ ". So we assume that $m>b_{5}$. In this case, we prove the lemma by contradiction. Suppose that

$$
\varrho_{D}(x, y)<2^{m-b_{5}} d_{D}(x)
$$

Then

$$
\begin{aligned}
d_{D}(y) & \leq \varrho_{D}(x, y)+d_{D}(x) \\
& <\left(2^{m-b_{5}}+1\right) d_{D}(x) \\
& \leq \frac{2^{m}}{\left(8 b_{4}+1\right)^{c_{2}}} d_{D}(x) \\
& <\frac{2^{m}}{\left(8 b_{4}+1\right)^{\frac{c_{2}}{2}}} d_{D}(x)
\end{aligned}
$$

By (2.1) and (2.13) we have

$$
d_{D}\left(y_{0}\right) \geq \frac{1}{2} d_{D}\left(x_{0}\right)=2^{m-1} d_{D}(x)>\frac{2^{m}}{\left(8 b_{4}+1\right)^{\frac{c_{2}}{2}}} d_{D}(x),
$$

then we obtain from $(2.27),(2.28)$ and the easy fact

$$
\frac{2^{m}}{\left(8 b_{4}+1\right)^{\frac{c_{2}}{2}}}=2^{m-\frac{c_{2}}{2} \log _{2}\left(8 b_{4}+1\right)}>1
$$


that there exist $w_{1} \in \gamma\left[x, x_{0}\right]$ and $w_{2} \in \gamma\left[y, y_{0}\right]$ such that

$$
d_{D}\left(w_{1}\right)=d_{D}\left(w_{2}\right)=\frac{2^{m}}{\left(8 b_{4}+1\right)^{\frac{c_{2}}{2}}} d_{D}(x) .
$$

On one hand, we obtain from (1.2), (2.1), (2.29), Theorems B and E that

$$
\begin{aligned}
c_{2} \log \left(1+\frac{\varrho_{D}\left(w_{1}, w_{2}\right)}{d_{D}\left(w_{1}\right)}\right) & \geq \widetilde{\alpha}_{D}\left(w_{1}, w_{2}\right) \\
& =\alpha_{D}\left(\gamma\left[w_{1}, w_{2}\right]\right) \\
& \geq \alpha_{D}\left(w_{1}, x_{m+1}\right)+\alpha_{D}\left(x_{m+1}, w_{2}\right) \\
& \geq 2 \log \frac{d_{D}\left(x_{m+1}\right)}{d_{D}\left(w_{1}\right)} \\
& \geq c_{2} \log \left(1+8 b_{4}\right),
\end{aligned}
$$

which imply that

$$
\varrho_{D}\left(w_{1}, w_{2}\right) \geq 8 b_{4} d_{D}\left(w_{1}\right) .
$$

On the other hand, by $(2.22),(2.23),(2.26)$ and (2.29) we obtain

$$
\begin{aligned}
\varrho_{D}\left(w_{1}, w_{2}\right) & \leq \varrho_{D}\left(w_{1}, x\right)+\varrho_{D}(x, y)+\varrho_{D}\left(y, w_{2}\right) \\
& \leq \operatorname{diam}\left(\gamma\left[x, w_{1}\right]\right)+\varrho_{D}(x, y)+\operatorname{diam}\left(\gamma\left[y, w_{2}\right]\right) \\
& <2 b_{4} d_{D}\left(w_{1}\right)+\varrho_{D}(x, y) \\
& \leq\left(2 b_{4}+\frac{2^{m-b_{5}}\left(8 b_{4}+1\right)^{\frac{c_{2}}{2}}}{2^{m}}\right) d_{D}\left(w_{1}\right) \\
& <\left(2 b_{4}+1\right) d_{D}\left(w_{1}\right)
\end{aligned}
$$

which is contradict with $(2.30)$. Hence the proof of the lemma is complete.

Now we are ready to conclude the proof of (2.25). It follows from (2.1), (2.13), (2.22), (2.23), (2.28), Lemmas 3 and 4 that

$$
\begin{aligned}
\operatorname{diam}(\gamma[x, y]) & \leq \operatorname{diam}\left(\gamma\left[x, x_{0}\right]\right)+\operatorname{diam}\left(\gamma\left[x_{0}, y_{0}\right]\right)+\operatorname{diam}\left(\gamma\left[y_{0}, y\right]\right) \\
& \leq 4 b_{4} d_{D}\left(x_{m+1}\right) \\
& =2^{m+2} b_{4} d_{D}(x) \\
& \leq 2^{b_{5}+2} b_{4} \varrho_{D}(x, y) .
\end{aligned}
$$

Hence the proof of (4) of Theorem 1 is complete by taking $c_{3}=2^{b_{5}+2} b_{4}$.

\subsection{The proof of Corollary 1}

$(1) \Rightarrow(2)$. Suppose (1) holds. Then by Theorem $H$, we see that $D$ is a $\mu$-uniform, so it is obvious inner uniform.

For the second part of (2), we can obtain easily from (1.1) and the definition of $A$-uniform (see Theorem H). Hence (2) is true. 
$(2) \Rightarrow(1)$. Suppose (2) holds. Then by Theorem 1, we know that for all $x, y \in D$,

$$
k_{D}(x, y) \leq c_{1} j_{D}^{\prime}(x, y) \leq c_{1} \mu_{5} \alpha_{D}(x, y),
$$

which shows that $D$ is $A$-uniform with coefficient $K=c_{1} \mu_{5}$.

Acknowledgements. The authors thank the referees who have made valuable comments on this manuscript.

\section{References}

[1] D. Barbilian, Einordnung von Lobayschewskys Massenbestimmung in einer gewissen allgemeinen Metrik der Jordansche Bereiche, Casopsis Mathematiky a Fysiky 64 (193435), 182-183.

[2] A. F. Beardon, The Apollonian metric of a domain in $\mathbb{R}^{n}$, in: Quasiconformal Mappings and Analysis, edited by Peter Duren, Juha Heinonen, Brad Osgood, and Bruce Palka (Springer-Verlag, New York), 91-108, 1998.

[3] M. Bonk, J. Heinonen, and P. Koskela, Uniformizing Gromov hyperbolic spaces, Asterisque $\mathbf{2 7 0}$ (2001), 1-99.

[4] F. W. Gehring, Uniform domains and the ubiquitous quasidisks, Jahresber. Deutsch. Math.-Verein. 89 (1987), no. 2, 88-103.

[5] F. W. Gehring and K. Hag, The Apollonian metric and quasiconformal mappings, In the tradition of Ahlfors and Bers (Stony Brook, NY, 1998), 143-163, Contemp. Math., 256, Amer. Math. Soc., Providence, RI, 2000

[6] F. W. Gehring and B. G. Osgood, Uniform domains and the quasihyperbolic metric, J. Analyse Math. 36 (1979), 50-74.

[7] F. W. Gehring and B. P. Palka, Quasiconformally homogeneous domains, J. Analyse Math. 30 (1976), 172-199.

[8] P. Hästö, The Apollonian inner metric, Comm. Anal. Geom. 12 (2004), no. 4, 927-947.

[9] _ The Apollonian metric: Uniformity and quasiconvexity, Ann. Acad. Sci. Fenn. Math. 28 (2003), no. 2, 385-414.

[10] - The Apollonian metric: limits of the comparison and bilipschitz properties, Abstr. Appl. Anal. 2003 (2003), no. 20, 1141-1158.

[11] - The Apollonian metric: quasi-isotropy and Seittenranta's metric, Comput. Methods Funct. Theory 4 (2004), no. 2, 249-273.

[12] sets, J. Appl. Anal. 12 (2006), no. 2, 209-232.

[13] M. Huang, S. Ponnusamy, X. Wang, and S. K. Sahoo, The Apollonian inner metric and uniform domain, Math. Nachr. 283 (2010), no. 9, 1277-1290.

[14] K. W. Kim, The quasihyperbolic metric and analogues of the Hardy-Littlewood property for $\alpha=0$ in uniformity John domains, Bull. Korean. Math. Soc. 43 (2006), no. 2, 395-410.

[15] - Inner uniform domains, the quasihyperbolic metric and weak bloch functions, Bull. Korean. Math. Soc. 49 (2012), no. 1, 11-24.

[16] O. Martio, Definitions for uniform domains, Ann. Acad. Sci. Fenn. Ser. A I Math. 5 (1980), no. 1, 197-205.

[17] O. Martio and J. Sarvas, Injectivity theorems in plane and space, Ann. Acad. Sci. Fenn. Ser. A I Math. 4 (1978), 383-401.

[18] R. Näkki and J. Väisälä, John disks, Exposition. Math. 9 (1991), no. 1, 3-43.

[19] A. G. Rhodes, An upper bound for the hyperbolic metric of a convex domain, Bull. London Math. Soc. 29 (1997), no. 5, 592-594.

[20] P. Seittenranta, Möbius-invariant metrics, Math. Proc. Cambridge Philos. Soc. 125 (1999), no. 3, 511-533. 
[21] J. Väisälä, Uniform domains, Tohoku Math. J. 40 (1988), no. 1, 101-118.

[22] _ Free quasiconformality in Banach spaces. I, Ann. Acad. Sci. Fenn. Ser. A I Math. 15 (1990), no. 2, 355-379.

[23] _ Free quasiconformality in Banach spaces. II, Ann. Acad. Sci. Fenn. Ser. A I Math. 16 (1991), no. 2, 255-310.

[24] Relatively and inner uniform domains, Conform. Geom. Dyn. 2 (1998), 56-88.

[25] M. Vuorinen, Conformal invariants and quasiregular mappings, J. Anal. Math. 45 (1985), 69-115.

\section{YAXIANG Li}

College of Science

Central South University of Forestry and Technology

Changsha, Hunan 410004, P. R. China

E-mail address: yaxiangli@163.com

Xiantao Wang

Department of Mathematics

HunAN NORMal University

Changsha, Hunan 410081, P. R. China

E-mail address: xtwang@hunnu.edu.cn 